\title{
Minimum mean square error estimator for mobile location using the pseudo-range TOA measurements
}

\author{
Jiyan Huang ${ }^{431^{*}}$, Peng Liư ${ }^{2}$, Mingquan $\mathrm{Lu}^{4}$, Baogen $\mathrm{Xu}^{3}$, Zhongchu Rao ${ }^{3}$, Yihe \\ $W^{3}{ }^{3}$, Chuan $\mathrm{Lu}^{1}$, and Hongyan Zhang ${ }^{1}$
}

${ }^{1}$ Dept. of Electronic Engineering, University of Electronic Science and Technology of China, Chengdu, China.

\author{
${ }^{2}$ Space Star Technology Co., Ltd, Beijing, China \\ ${ }^{3}$ Tong Fang Electronic Technology Company Limited, Jiangxi, China \\ ${ }^{4}$ Tsinghua University, BeiJing, China \\ *Jiyan Huang is the corresponding author.huangjiyan@uestc.edu.cn
}

Keywords: Mobile location, Cramer-Rao Lower Bound (CRLB), non-synchronous mobile system, time difference of arrival (TDOA), pseudo-range TOA.

\begin{abstract}
Source Localization in the non-synchronous mobile system is a very important issue since it only requires synchronized clocks between the based stations (BSs) and can reduce system complexity. There are time-difference-of-arrival (TDOA) and pseudo-range time-of-arrival (TOA) based localization techniques for a non-synchronous mobile system. Although many methods and performance analysis have been proposed for TDOA technique, relatively few studies have been reported on pseudo-range TOA method. This paper proposes a novel localization algorithm based on minimum mean square error estimator (MMSEE) for pseudo-range TOA. The simulations show that the proposed method can reach its CRLB and has the better performance than the TDOA method based on maximum likelihood estimator (MLE).
\end{abstract}

\section{Introduction}

Location estimation of a mobile station (MS) in wireless communication systemshas gained considerable attention since the Federal Communication Commission passed a mandate requiring cellular providers to generate accurate location estimates for Enhanced-911 services [1]. Conventional geolocation techniques include time-of-arrival (TOA), time-difference-of-arrival (TDOA), angle-of-arrival (AOA), signal strength (SS) based methods, or a combination of these. Among these location techniques, methods based on TOA and TDOA have attracted much attention due to its high location accuracy [2-3]. The main problem of TOA localization technique is time synchronization. TOA technique uses the absolute time of arrival at a certain base station which requires accurate time synchronization between MS and based stations (BSs). It is almost impossible for a MS to finish such high precision time synchronization with the existing hardware configuration.

To overcome the imperfect time synchronization in MS, a non-synchronous mobile location system was developed and widely used in cellular location system and global positioning system. Compared with the TOA technique, the non-synchronous mobile location system only requires synchronized clocks between the BSs, and this characteristic reduces system complexity. Besides the unknown coordinates of MS in synchronous mobile system, there is an additional unknown parameter, i.e., the time offset between the clock at a MS and those at BSs, in a non-synchronous mobile system. The most popular localization method in non-synchronous mobile system is TDOA technique, which eliminates the time offset via linealized least squares using equations obtained by differencing between BSs. Many TDOA location methods have been proposed [4-7]. Chan and Ho [7] propose the two-step weighted least squares (WLS) method based on maximum likelihood estimator (MLE) to improve the location accuracy of the SI method. This method is an unbiased, and can provide an optimum performance and attain the deterministic Cramer-Rao lower bound 
(CRLB) asymptotically.

Although many methods and performance analysis have been proposed for TDOA technique, relatively few studies have been reported on another location technique in non-synchronous mobile system called pseudo-range TOA. Compared with the TDOA eliminating the time offset by differencing equations between BSs, the pseudo-range TOA method treated the time offset as an unknown parameter and tried to estimate it. In this paper, a novel pseudo-range TOA method based on minimum mean square error estimator (MMSEE) for non-synchronous mobile system is proposed. Since statistical characteristics of time-offset are used as prior information, the proposed method can provide the better performance than TDOA method based on MLE.

\section{System Model}

Firstly, the basic system model of a non-synchronous mobile system is briefly introduced. Besides the unknown coordinates of MS, there is an additional unknown parameter, i.e., the time offset between the clock at a MS and those at BSs, in a non-synchronous mobile system. There are, broadly, two ways to cope with the time offset: processing pseudo-range measurements directly (pseudo-range TOA method) via linearized least squares or via linealized least squares using equations obtained by differencing between BSs (TDOA method). Consider a non-synchronous mobile system, let $N=\{1, \cdots, \mathrm{N}\}$ be the set of indices of the BSs, whose locations $\left\{\left(x_{i}, y_{i}\right)^{T}, i \in N\right\}$ are known. The unknown parameters are the MS position $(x, y)$ and time-offset $\tau$. The delay time $t_{i}$ is the transmission time between MS and BS $i$. Denote the measurement with noise of $\{*\}$ as $\{\widetilde{*}$. A delay estimate can be approximated as:

$$
\bar{t}_{i}=t_{i}+n_{i} \text {, for } i \in N
$$

The delay $t_{i}$ is called as the pseudo-range delay by using the pseudo-range concept:

$$
t_{i}=\frac{1}{c}\left(d_{i}+1\right), \text { for } i \in N
$$

with $n_{i}$ is a zero mean Gaussian random variable with variance $\sigma_{i}^{2}, c$ is the signal propagation speed, $l=c \tau$ is the pseudo-range caused by time-offset $\tau$, and $d_{i}$ is the true distance between the BS $i$ and MS.

$$
d_{i}=\sqrt{\left(x_{i}-x\right)^{2}+\left(y_{i}-y\right)^{2}}
$$

The corresponding pseudo-range measurement $\hat{F}_{i}$ is model as:

$$
\hat{r}_{i}=c \bar{t}_{i}=d_{i}+1+c n_{i}
$$

The covariance matrix of $\hat{r}_{i}$ can be easily calculated from (4):

$$
Q_{p}=c^{2}\left[\begin{array}{ccc}
\sigma_{1}^{2} & \cdots & 0 \\
\vdots & \ddots & \vdots \\
0 & \cdots & \sigma_{N}^{2}
\end{array}\right]
$$

The TDOA data can be obtained from (1):

$$
\begin{aligned}
& \bar{t}_{i 1}=\bar{t}_{i}-\bar{t}_{1}=\left(t_{i}-t_{1}\right)+\left(n_{i}-n_{1}\right) \\
& =\left(n_{i}-n_{1}\right)+\frac{1}{c}\left(d_{i}-d_{1}\right) \\
& \text { for } i=2, \ldots, N
\end{aligned}
$$

The range difference measurement $\hat{r}_{i 1}$ from the corresponding TDOA measurement can be calculated as:

with the covariance matrix

$$
\hat{r}_{i 1}=c\left(n_{i}-n_{1}\right)+\left(d_{i}-d_{1}\right)
$$




$$
Q_{D}=c^{2}\left[\begin{array}{ccc}
\sigma_{2}^{2}+\sigma_{1}^{2} & \cdots & \sigma_{1}^{2} \\
\vdots & \ddots & \vdots \\
\sigma_{1}^{2} & \cdots & \sigma_{N}^{2}+\sigma_{1}^{2}
\end{array}\right]
$$

The coordinates of MS can be estimated by either (a) using $N$ pseudo-range TOA measurements $\bar{t}_{i}$, whose errors are independent, and solving for 3 unknowns $(x, y, l)$ (pseudo-range TOA method), or using $N-1$ TDOA measurements $\hat{t}_{i 1}$, whose errors are dependent, and solving for 2 unknowns $(x, y)$ (TDOA method). This paper focuses on the pseudo-range TOA method.

\section{Minimum mean square error estimator for mobile location using the pseudo-range TOA measurements}

Mobile location in a non-synchronous mobile system using TDOA measurements has been widely studied in the literature. Among the existing TDOA location methods, the method proposed by Chan and Ho [7] based on MLE hasattractedmuchattention since it is an unbiased, and can provide an optimum performance. There are two ways for the pseudo-range TOA method based on different assumptions on the pseudo-range: $l$ is the deterministic or $l$ is modeled as a Gaussian random variable with variance $\sigma_{l}^{2}$. The first method evaluates the root mean square error (RMSE) from the average value of the observed measurements such as pseudo-range TOA, while the latter provides the RMSE from both average value of the observed measurements and probability density function (PDF) of the pseudo-range $l$. Therefore the latter has the minimum RMSE "on the average”, i.e., with respect to all the pseudo-range realizations.

This implies that it is not possible for a non-synchronous mobile system to further reduce the RMSE using the pseudo-range TOA measurements based on the assumption that $l$ is the deterministic unknown parameter. This also means the positioning accuracy of a non-synchronous mobile system can be further improved through using pseudo-range TOA measurements and modeling $l$ as a Gaussian random variable. This section proposes a MMSEE for the pseudo-range TOA based location method. Since statistical characteristics of time-offset are used as prior information, the proposed method can provide the better performance than Chan's method based on MLE.

From (4), the pseudo-range $r_{i}$ can be modeled as:

$$
r_{i}=d_{i}+1=\sqrt{\left(x-x_{i}\right)^{2}+\left(y-y_{i}\right)^{2}}+1
$$

The square distance between the MS and BS $\mathrm{i}$ is:

$$
\left(r_{i}-1\right)^{2}=r_{i}^{2}-2 r_{i} 1+l^{2}=\left(x-x_{i}\right)^{2}+\left(y-y_{i}\right)^{2}
$$

The above equation at $\mathrm{i}=1$ becomes:

$$
r_{1}^{2}-2 r_{1} l+l^{2}=\left(x-x_{1}\right)^{2}+\left(y-y_{1}\right)^{2}
$$

Subtracting (11) from (10), gives:

$$
\begin{aligned}
& r_{i}^{2}-r_{1}^{2}-\left(K_{i}-K_{1}\right) \\
& =-2 x\left(x_{i}-x_{1}\right)-2 y\left(y_{i}-y_{1}\right)+2 l\left(r_{i}-r_{1}\right)
\end{aligned}
$$

where $\mathrm{K}_{\mathrm{i}}=x_{i}^{2}+y_{i}^{2}$.

Let $Z=\left[\begin{array}{lll}Z_{1} & Z_{2} & Z_{3}\end{array}\right]^{T}=\left[\begin{array}{lll}x & y & 1\end{array}\right]^{T}$. With the measurement noise, the error vector derived from (12) is:

where,

$$
\mathrm{e}=\mathrm{Y}-\mathrm{GZ}(13)
$$




$$
\begin{aligned}
& \mathrm{G}=\left[\begin{array}{ccc}
-\left(\mathrm{x}_{2}-x_{1}\right) & -\left(\mathrm{y}_{2}-\mathrm{y}_{1}\right) & \widehat{r}_{2}-\widehat{r}_{1} \\
\vdots & \vdots & \vdots \\
-\left(\mathrm{x}_{\mathrm{N}}-x_{1}\right) & -\left(\mathrm{y}_{\mathrm{N}}-\mathrm{y}_{1}\right) & \widehat{r}_{2}-\widehat{r}
\end{array}\right], \\
& \mathrm{Y}=\frac{1}{2}\left[\widehat{r}_{2}^{2}-\widehat{r}_{1}^{2}-K_{2}+K_{1} \quad \cdots \quad \widehat{r}_{N}^{2}-\widehat{r}_{1}^{2}-K_{N}+K_{1}\right]^{T}, \mathrm{~K}_{\mathrm{i}}=x_{i}^{2}+y_{i}^{2} .
\end{aligned}
$$

The MLE of $Z$ is:

$$
\widehat{Z}=\left(G^{\mathrm{T}} \psi^{-1} G\right)^{-1} G^{T} \psi^{-1} Y
$$

The MMSEE [18] of $Z$ in the proposed method is:

$$
\widehat{Z}=\left(Q_{\theta}+G^{\mathrm{T}} \psi^{-1} G\right)^{-1} G^{T} \psi^{-1} Y
$$

where $\psi$ is the covariance matrix of $\mathrm{e}$ :

where $Q_{\theta}=\operatorname{diag}\left(\left[\left[\begin{array}{lll}0 & 0 & \sigma_{l}^{-2}\end{array}\right]\right)\right.$.

$$
\psi=\operatorname{cov}(e)=E\left(e e^{T}\right)
$$

Unlike the MLE in Chan's method, the MMSEE in (15) utilizes the statistical characteristics $Q_{\theta}$ of time-offset as prior information to improve the positioning accuracy.

From (13), the element $e_{i}$ of e can be expressed as:

$$
\begin{aligned}
\mathrm{e}_{\mathrm{i}} & =\frac{1}{2}\left(\hat{r}_{i}^{2}-\hat{r}_{1}^{2}-\left(K_{i}-K_{1}\right)\right) \\
& +\mathrm{x}\left(x_{i}-x_{1}\right)+y\left(y_{i}-y_{1}\right)-l\left(\hat{r}_{i}-\widehat{r}_{1}\right)
\end{aligned}
$$

Substituting (4) into (17) and ignoring the square error term, gives:

$$
\mathrm{e}_{\mathrm{i}}=\left(r_{i}-1\right) c n_{i}-\left(r_{1}-1\right) c n_{1}(18)
$$

Substituting (18) into $\mathrm{e}_{\mathrm{i}} e_{j}$, gives:

$$
\begin{aligned}
\mathrm{e}_{\mathrm{i}} e_{j}= & \left(r_{i}-1\right)\left(r_{j}-1\right) c^{2} n_{i} n_{j}-\left(r_{i}-1\right)\left(r_{1}-1\right) c^{2} n_{i} n_{1} \\
& -\left(r_{1}-1\right)\left(r_{j}-1\right) c^{2} n_{1} n_{j}+\left(r_{1}-1\right)^{2} c^{2} n_{1}^{2}
\end{aligned}
$$

When, $i=j$,

$$
\begin{aligned}
& \mathrm{e}_{\mathrm{i}}^{2}=\left(r_{i}-l\right)^{2} c^{2} n_{i}^{2}-2\left(r_{i}-l\right)\left(r_{1}-l\right) c^{2} n_{i} n_{1} \\
& +\left(r_{1}-l\right)^{2} c^{2} n_{1}^{2}
\end{aligned}
$$

From (19) and (20), the expectations of $\mathrm{e}_{\mathrm{i}} e_{j}$ and $\mathrm{e}_{\mathrm{i}}^{2}$ can be obtained:

$$
\begin{aligned}
& \mathrm{E}\left(\mathrm{e}_{\mathrm{i}} e_{j}\right)=\left(r_{1}-l\right)^{2} c^{2} \sigma_{1}^{2} \\
& E\left(e_{i}^{2}\right)=\left(r_{i}-l\right)^{2} c^{2} \sigma_{i}^{2}+\left(r_{1}-l\right)^{2} c^{2} \sigma_{1}^{2}
\end{aligned}
$$

Substituting (21) into (16), gives:

$$
\psi=\operatorname{cov}(\mathrm{e})=\mathrm{E}\left(\mathrm{ee}^{\mathrm{T}}\right)=\psi_{1}+\psi_{2}
$$

where, $\psi_{1}=c^{2} \operatorname{diag}\left\{\left[\left(r_{1}-l\right)^{2} \sigma_{1}^{2} \quad \ldots \quad\left(r_{N}-l\right)^{2} \sigma_{N}^{2}\right]\right\}, \psi_{2}=\left(r_{1}-l\right)^{2} c^{2} \sigma_{1}^{2} 1_{(N-1) \times(N-1)}$, and $1_{i \times \mathrm{j}}$ is $(i \times j)$-dimensioned matrix constituted of ones.

In fact, $\psi$ is unknown as $\psi$ contains the unknown $r_{1}$ and 1 . Further approximation is necessary in order to make the problem solvable. When MS is far from the BSs, $\left(r_{i}-1\right)^{2} \sigma_{i}^{2} \approx(r-1)^{2} \sigma^{2}$ and $\psi$ can be simplified as:

$$
\psi=(r-I)^{2} \sigma^{2}\left(I+1_{(N-1) \times(N-1)}\right)=(r-I)^{2} \sigma^{2} Q
$$

where, $Q=I+1_{(N-1) \times(N-1)}$.

For weighted matrix $\psi$ in (22), the effect of scaling factor $(r-l)^{2} \sigma^{2}$ can be ignored. Thus, an approximation of (14) is: 


$$
\widehat{Z}=\left(G^{T} Q^{-1} G\right)^{-1} G^{T} Q^{-1} Y \quad(24)
$$

The approximate value $\hat{r}_{i}$ and $\hat{Z}_{3}$ obtained from (24) can be used in $\psi$ to make the MMSEE of $Z$ (15) solvable.

It should be noted that the unknown parameters $(x, y, l)$ in $Z$ are independent whereas those of Chan's method are related. Unlike Chan's method, it is not necessary to perform the second step weighted solution in the proposed method.

\section{Simulation Results}

The simulations are based on the cells of $2000 \mathrm{~m}$ of diameter. A hexagonal test cell is surrounded by 6 neighboring cells. The coordinates of BSs are $\mathrm{BS}_{1}(0,0) \mathrm{m}, \mathrm{BS}_{2}(1732,1000) \mathrm{m}, \mathrm{BS}_{3}(0,2000) \mathrm{m}$, $\mathrm{BS}_{4}(-1732,1000) \mathrm{m}, \mathrm{BS}_{5}(-1732,-1000) \mathrm{m}, \mathrm{BS}_{6}(0,-2000) \mathrm{m}$, and $\mathrm{BS}_{7}(-1730,-1000) \mathrm{m}$. The position of MS is uniformly distributed in the square space $-1000 \leq x, y \leq 1000 \mathrm{~m}$.

The RMSEs are defined as $\sqrt{E\left[(x-\widehat{x})^{2}+(y-\hat{y})^{2}\right]}$ in the units of $\mathrm{m}$, and are obtained from the average of 5000 independent runs. To compare with the proposed method, the Chan's method [7] based on MLE and TDOA measurements is selected here since it has the optimum performance and canattain the CRLB of TDOA asymptotically. CRLBs of the Pseudo-Range TOA and TDOA are also compared in the simulation.

Fig. 1 is simulated to show the performance of the proposed method in different noise environments. The std of time measurement noise is varied from $0.667 \times 10^{-7} \mathrm{~S}$ to $3.333 \times 10^{-7} \mathrm{~S}$ and the std of pseudo-rang is $30 \mathrm{~m}$. It can be observed that both of Chan's method and the proposed method can attain the corresponding CRLBs. Since the prior information on time offset is used in the proposed method, it has the higher location accuracy than Chan's method as shown in Fig. 1.

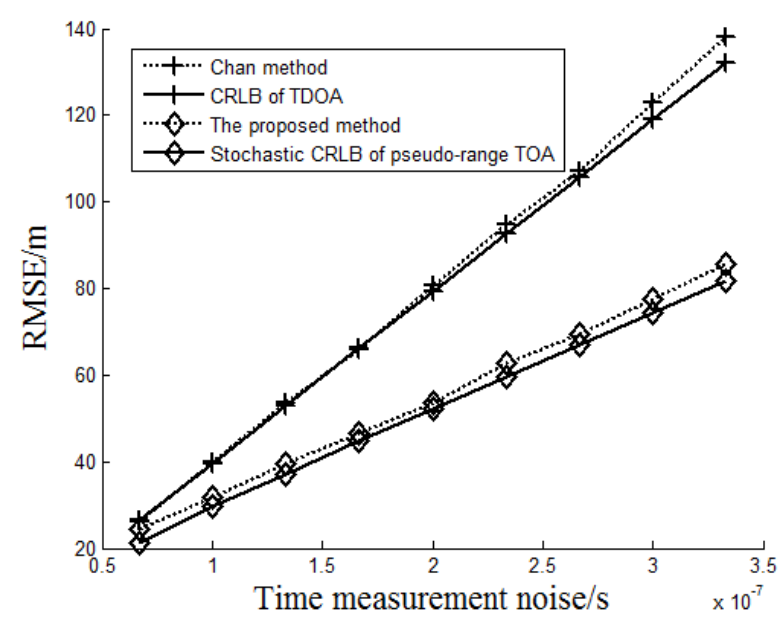

Fig. 1 Comparisons among the proposed method, Chan's method, and CRLBs

\section{Conclusions}

Both TDOA and pseudo-range TOA methods can be used for a non-synchronous mobile location system. Compared with the wide application of TDOA method, relatively few studies have been reported on pseudo-range TOA method. In this paper, a novel localization algorithm using MMSEE is proposed for pseudo-range TOA. The simulations show that the proposed method can reach its CRLB and performs better than the TDOA method.

\section{Acknowledgements}

This work was supported by the Open Research Fund of The Academy of Satellite Application under grant (2014_CXJJ-DH_09), the National Natural Science Foundation of China (61201275), 
the Fundamental Research Funds for the Central Universities (ZYGX2013J026), China Postdoctoral Science Foundation (2013M540532), and Jiangxi Postdoctoral Research Project (2013KY01). The authors would like to thank the anonymous referees for their suggestions and comments.

\section{References}

[1] FCC.: 'FCC Acts to Promote Competition and Public Safety in Enhanced Wireless 911 Services’, Washington, DC: WT Rep, September 15, 1999,pp. 99-27

[2] Caffery, J.J., and Stuber, G.L.: 'Overview of radiolocation in CDMA cellular systems', IEEE Commun. Mag., 1998, 36, (4), pp. 38-45

[3] Drane, C., Macnaughton, M., and Scott, C.: 'Positioning GSM telephones', IEEE Commun. Mag., 1998, 36, (4), pp. 46-59

[4] He-Wen Wei, Rong Peng, Qun Wan, Zhang-Xin Chen, Shang-Fu Ye, "Multidimensional Scaling Analysis for Passive Moving Target Localization With TDOA and FDOA Measurements," IEEE Transactions on Signal Processing, vol. 58, no. 3, pp. 1677 - 1688, 2010.

[5] J.S.Abel,“A divide and conquer approach to least-squares estimation,”IEEE Trans. Aerosp. Electron. Syst., vol. 26, no. 2, pp. 423-427, Feb. 1990.

[6] B. Friedlander,“A passive localization algorithm and its accuracy analysis,” IEEE J. Ocean. Eng.,vol. 12, no. 1, pp. 234-245, Jan. 1987.

[7] Y.T.Chan, and K.C.Ho,“A simple and efficient estimator for hyperbolic location,”IEEE Trans. Signal Process., vol. 42, no. 8, pp. 1905-1915, Aug. 1994. 